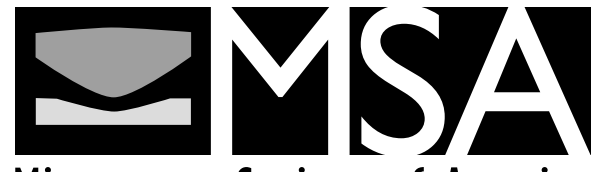

Microscopy Society of America

Membership Application
Please fill out this form and submit it via fax or U.S. mail, or complete the form online at www.microscopy.org. You may also use this form to reinstate your membership or update your personal records with MSA.

Toll Free 1. 800. 538. 3672 Phone 703. 234. $4115 \mid$ Fax 703. 435. 4390

E-Mail BusinessOffice@microscopy.org

Mail form and payment to:

Microscopy Society of America

C/O Wachovia Bank

Lockbox \# 759225 Baltimore, MD 21275-9225

\title{
Personal Information
}

\begin{tabular}{llcc}
\hline Prefix & First Name & Ml & Last Name \\
\hline E-Mail & & Corporate / Institutional Web Site (required for Sustaining Members) & Title \\
\hline Organization Name & & This is my: $\square$ Home Address \\
\hline Preferred Mailing Address & & State & ZIP \\
\hline City & & Country
\end{tabular}

Phone

Fax

One-Year Dues (January 1 to December 31)

Regular (Open to anyone with a professional interest in microscopy and related areas)

North America (USA, Canada, Mexico)

Outside North America

$\square 60.00$

$\square \$ 78.00$

Student (Open to bona fide full-time students) North America (USA, Canada, Mexico)

Outside North America

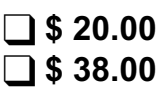

Sustaining (Company-based)

North America (USA, Canada, Mexico)

Outside North America

Focused Interest Groups (FIGs) - Optional (Add \$10.00 each)

$\square$ Atom Probe Field Ion Microscopy $\square$ Biomaterials $\square$ Cryopreparation

$\square$ Facilities Operations \& Management $\square$ Focused Ion Beam

$\square$ Materials Research in an Aberration-Free Environment $\square$ Pharmaceuticals

Vascular Corrosion Casting

Public Policy Support - Optional (Tax Deductible)

$\square \$ 20.00 \square \$ \mathbf{3 0 . 0 0} \square \mathbf{5 0 . 0 0} \square$ Other \$

Additional Journal Subscriptions - Optional (Based on 2008 Rates)

$\square$ Journal of Electronic Microscopy

$\square$ Journal of Microscopy

$\square$ Materials Characterization

$\square$ Micron

$\square$ Microscopy Research \& Technique

$\square$ Ultramicroscopy
$\$ 210.00$

$\$ 230.00$

$\$ 175.00$

Outside North America

$\$ 185.00$

$\$ 185.00$

$\$ 325.00$

Outside North America

$\$ 415.00$

$\$ 360.00$

Continued $\triangleright$ 


\section{Payment Information}

$\square$ VISA $\square$ MasterCard $\square$ AMEX $\square$ Check

Name as it appears on credit card

Card Number

Expiration Date

Signature

Phone

\section{Fee Calculation}

Annual Dues

Focused Interest Groups

Public Policy Support

Journal Subscriptions

TOTAL PAYMENT
$\$$

$\$$

$\$$

$\$$

$\$$
Multiple year renewal is available with online application.

Go to: www.microscopy.org

for more information

NOTE: Please allow up to two weeks for processing.

Fields of Microscopy/Microanalysis You Use in Your Work (Check all that apply)

$\begin{array}{llll}\square \text { Light / Optical } & \square \text { Confocal } & \square \text { P Photon } & \square \text { Near Field } \\ \square \text { IR / Raman } & \square \text { Acoustic } & \square \text { Scanning Probe } & \square \text { Scanning lon } \\ \square \text { X-Ray Microscopy } & \square \text { SEM } & \square \text { TEM } & \square \text { STEM } \\ \square \text { IVEM / HVEM } & \square \text { LEEM } & \square \text { AEM } & \square \text { EELS } \\ \square \text { EDS } & \square \text { WDS } & \square \text { EMPA } & \square \text { AES } \\ \square \text { ESCA } & \square \text { SIMS } & \square \text { FIM / AtomProbe } & \square \text { FIB } \\ \square \text { MRI } & \square \text { XRF / XRD } & \square \text { EBSD } & \end{array}$

Membership in Other Societies (Check those to which you are a member)
$\square$ AAAS (American Association for the Advancement of Science)
$\square$ AHA (American Heart Association
AIP (American Institute of Physics)
$\square$ AMA (American Medical Association)
$\square$ APS (American Physical Society)
$\square$ ASCB (American Society of Cell Biology)
$\square$ ASIP (American Society of Investigative Pathology)

\footnotetext{
$\square$ EMAG (Institute of Physics Electon Microscopy \& Analysis Group)

$\square$ HS (Histochemical Society)

$\square$ IMS (International Metallographic Society)

$\square$ MNZ (Microscopy New Zealand)

$\square$ MRS (Materials Research Society)

$\square$ MSC (Microscopical Society of Canada)

$\square$ OSA (Optical Society of America)

$\square$ SPIE (International Society of Optical Engineering)
} 


\begin{tabular}{ll}
\hline INDEX TO ADVERTISERS & \\
\hline Advanced Microscopy Techniques, AMT & A16 \\
Brüker AXS & A11 \\
Diatome & A5, 376 \\
Electron Microscopy Sciences, EMS & A17 \\
Gatan & A6 \\
JEOL & A3, Cover 4 \\
Leica Microsystems & Cover 2 \\
2009 MSA Application Form & A13, A14 \\
Micro Star Diamond Knives & A18 \\
Omniprobe & Cover 3 \\
Soft Imaging Solution & A15 \\
SPI Supplies & A10 \\
Ted Pella & A12, 375 \\
Thermo Scientific & A9 \\
Tousimis & 374 \\
\end{tabular}

